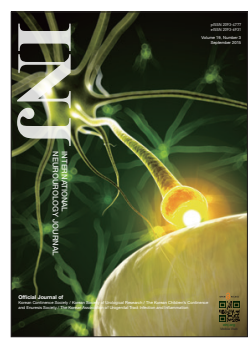

\title{
Implantable Bladder Sensors for Long-term Monitoring of Bladder Volume
}

\author{
Su Jin Kim \\ Department of Urology, The Catholic University of Korea School of Medicine, Seoul, Korea \\ E-mail: hygeia@catholic.ac.kr
}

Voiding diaries, also called frequency volume charts, are an essential instrument to explore the voiding status of patients with lower urinary tract symptoms (LUTS) and incontinence [1]. Patients record micturition times, voided volume with or without other factors including fluid intake, incontinence, urgency, and urge incontinence over a period of days. This task is associated with patient burden, and increased recording periods decrease patient compliance [2]. Although several electronic voiding diaries have recently been developed and applied to decrease patient burden, the problem persists. Small sensors implanted in the bladder to automatically record pressure and volume and times of voiding may offer a new option for assessment and treatment of LUTS and incontinence.

Several studies have described the development of implantable bladder sensors to monitor real-time functional changes [3,4]. In this issue of International Neurourology Journal, Dakurah et al. [5] introduce several types of implantable bladder sensors and discuss specific issues regarding hermeticity, biocompatibility, telemetry, drift, power transfer, and compatibility with medical imaging tools such as computed tomography and magnetic resonance imaging. Although an unmet clinical need might enable medical device development, we clinicians should understand from an engineering viewpoint the possible improvements and emerging trends in the design of implantable bladder sensors that may overcome the current issues [6].

- Conflict of Interest: No potential conflict of interest relevant to this article is reported.

\section{REFERENCES}

1. Abrams P, Klevmark B. Frequency volume charts: an indispensable part of lower urinary tract assessment. Scand J Urol Nephrol Suppl 1996;179:47-53.

2. Ku JH, Jeong IG, Lim DJ, Byun SS, Paick JS, Oh SJ. Voiding diary for the evaluation of urinary incontinence and lower urinary tract symptoms: prospective assessment of patient compliance and burden. Neurourol Urodyn 2004;23:331-5.

3. Mendez A, Belghith A, Sawan M. A DSP for sensing the bladder volume through afferent neural pathways. IEEE Trans Biomed Circuits Syst 2014;8:552-64.

4. Melgaard J, Rijkhoff NJ. Detecting the onset of urinary bladder contractions using an implantable pressure sensor. IEEE Trans Neural Syst Rehabil Eng 2011;19:700-8.

5. Dakurah MN, Koo C, Choi W, Joung YH. Implantable bladder sensors: a methodological review. Int Neurourol J 2015;19:133-41.

6. Joung YH. Development of implantable medical devices: from an engineering perspective. Int Neurourol J 2013;17:98-106. 